\title{
Propuesta de un nuevo enfoque para reducir el abandono escolar en secundaria
}

\author{
New Approach to reduce High School Dropout Rates
}

\author{
Mauricio Cristhian Portillo-Torres ${ }^{1}$ \\ Ministerio de Educación Pública \\ San José, Costa Rica \\ portillozeledon@gmail.com
}

Recibido 3 de setiembre de 2014 • Corregido 16 de enero de 2015 • Aceptado 27 de abril de 2015

\begin{abstract}
Resumen. Durante el período 2006-2014, el Ministerio de Educación Pública de Costa Rica implementó cuatro acciones estratégicas para la disminución del abandono escolar en secundaria. El objetivo principal de estas acciones fue promover la participación estudiantil y la identificación del estudiante con su centro educativo. Para valorar el impacto de estas acciones se revisaron estudios elaborados por el Ministerio de Educación y la Contraloría General de la República. A partir de los resultados se ve la necesidad de proponer una visión más integral para lograr la permanencia estudiantil en secundaria con base al concepto de engagement. Así mismo se propone un modelo de acciones estratégicas que incluye acciones preventivas universales, acciones preventivas selectivas y acciones preventivas dirigidas.
\end{abstract}

Palabras claves. Abandono escolar, deserción escolar, participación estudiantil

Abstract. From 2006 to 2014, the Ministry of Public Education of Costa Rica implemented four strategic actions to reduce high school dropout rates. The main purpose of these actions was to promote student participation and student identification with their school. Studies prepared by the Ministry of Education and the Comptroller of the Republic were revised to assess the impact of these actions. The result of these actions does not show an actual decrease in the number of students who leave high school. Therefore, a more holistic view is necessary to ensure the students' stay. This review suggests using use the concept of student engagement and applying a three tier system-wide dropout preventive actions: universal, targeted and intensive.

Keywords. Dropping out, dropouts, student engagement.

\footnotetext{
${ }^{1}$ Licenciado en Docencia de la Teología (UNED) y Master en Educación por el Instituto Tecnológico de Monterrey. Actualmente trabaja como asesor en la Dirección de Desarrollo Curricular del Ministerio de Educación Pública. Anteriormente ejerció como docente en diferentes colegios privados. Ha escrito y dado conferencias sobre diversos temas educativos, especialmente sobre el tema del abandono escolar. En el 2013 ganó el IV Concurso sobre deserción escolar del BID (Graduate XXI).
} 
doi: http://dx.doi.org/10.15359/ree.19-2.17

URL: http://www.una.ac.cr/educare

CORREO: educare@una.cr

En Costa Rica la educación básica es obligatoria y gratuita desde preescolar hasta completar todos los ciclos de la educación media. En este sentido, se ha logrado una universalidad del acceso a la educación en primaria. Sin embargo, en secundaria la tasa de escolaridad neta del III Ciclo (7०, $8^{\circ}$ y $9^{\circ}$ año) es del $87,8 \%$ y en educación diversificada $\left(10^{\circ}, 11^{\circ}\right.$ y $12^{\circ}$ año) es del $48 \%$ (Programa Estado de la Nación, 2013). Por tanto, el sistema educativo costarricense solo atiende a la mitad de jóvenes en edad de asistir al cuarto ciclo educativo.

El proceso de desescolarización de estudiantes costarricenses comienza a partir de los 13 años, un fenómeno similar a otros países latinoamericanos (Unicef, 2012). Como consecuencia, el 76,8\% del total de estudiantes que abandonaron sus estudios en el 2013 estaba matriculado en alguna modalidad de la secundaria tradicional (académica diurna, técnica diurna, académica nocturna, técnica nocturna). Es decir, 35414 estudiantes abandonaron las aulas durante el curso lectivo 2013, una cifra, en términos absolutos, similar a los años 2010 y 2004 (MEP, Departamento de Análisis Estadístico, 2014).

Las causas del abandono escolar costarricense han sido estudiadas en pequeños contextos (colegios, regiones), pero aún está pendiente una investigación que abarque todo el sistema educativo. Con base en los estudios exploratorios hechos por el Ministerio de Educación Pública, como el realizado por Jiménez y Gaete (2013), se han identificado y agrupado tres grandes grupos de causas: expulsión, repulsión y exclusión. La expulsión agrupa las causas generadas por el mismo sistema educativo como el mecanismo de evaluación; la repulsión se refiere a los motivos por falta de pertinencia o interés de la materia por la parte estudiantil; la exclusión son las razones asociadas a factores socio-económicos de vulnerabilidad.

El artículo recogerá tres estrategias que durante el período 2006-2014 el Ministerio de Educación Pública implementó para disminuir la exclusión estudiantil. El objetivo del artículo es mostrar el resultado de estas estrategias, el modelo preventivo subyacente, a fin de proponer una nueva forma de combatir el abandono escolar en secundaria.

\section{Acciones que buscan la participación e identificación}

Las tres acciones estratégicas implementadas por el Ministerio de Educación Pública durante el período 2006-2014 responden a dos de los grandes grupos de factores asociados con el abandono escolar (expulsión, repulsión). Estas acciones son:

- Reformas curriculares para que los contenidos sean permitentes y con metodologías de enseñanza más dinámicas

- Reforma a los métodos de evaluación

- Fortalecimiento de actividades extracurriculares a fin de promover la participación estudiantil 
doi: http://dx.doi.org/10.15359/ree.19-2.17

URL: http://www.una.ac.cr/educare

Las reformas curriculares tuvieron como referencia, en primer lugar, el proyecto educativo Ética, estética y ciudadanía, cuyo propósito fue desarrollar un enfoque curricular de tipo constructivista y socio-constructivista a fin de enseñar a valorar y disfrutar lo verdadero como lo bueno y lo bello, así como a saber convivir (MEP, 2014). Para tal fin el Misterio de Educación Pública contrató a la empresa Procesos que lideró y coordinó el diseño del nuevo currículo para la Educación Media en las siguientes materias: Educación Cívica, Educación Musical, Artes Plásticas, Educación Física, Vida Cotidiana (anteriormente Educación para el Hogar) y Artes Industriales (MEP, 2014).

Los nuevos programas incorporaron del constructivismo el papel activo del estudiantado, el cual construye su propio conocimiento a partir de la interacción con en medio físico y social. Esto lo hace con un sentido crítico y reflexivo. Así mismo se enfatizó que el aprendizaje se da cuando la persona relaciona lo que vive con sus experiencias y conocimientos previos. El rol docente propuesto es de mediador facilitador. Del enfoque socio-constructivista se incorporó que el alumnado participe como una ciudadanía en la vida social y que el currículo sirva como medio de conocimiento de la realidad y la problemática de la comunidad. Se enfatizó en la vivencia de valores ético, morales, estéticos y ciudadanos para vivir en una sociedad pluriétnica y pluricultural (MEP, 2009a).

A principios del 2008 se concluyó la elaboración del programa de Educación Cívica, posteriormente, el de Educación Musical. Ambos se empezaron a utilizar para el curso lectivo 2009. El programa de Artes Plásticas fue aprobado por el Consejo Superior de Educación en el 2009 y en el caso de Educación Física, en el 2010. Educación para la Vida Cotidiana y Artes Industriales fueron los últimos en ser aprobados en el año 2012 y 2013 respectivamente (MEP, 2014). Los cambios de programas de estas seis materias se acompañaron de una reforma parcial al reglamento de evaluación en el año 2009. Se cambiaron los porcentajes de los componentes de la calificación y se añadió el rubro de proyecto.

En el caso de Educación Musical, Artes Plásticas, Educación Física, Educación para el Hogar y Artes Industriales, los rubros de evaluación quedaron de la siguiente manera: trabajo cotidiano un $20 \%$ (III ciclo) o 10\% (Diversificado), trabajo extraclase 5\%, una prueba $25 \%$, el proyecto de un valor de 40\% (III ciclo) o 50\% (Diversificado), concepto 5\% y asistencia 5\% (MEP, 2009a).

Al ser las reformas de los programas un proceso progresivo, se cambiaron los valores de los rubros de Artes Industriales al aprobarse los nuevos programas en el 2013. Estos son los porcentajes de los rubros que aparecen en el programa: trabajo cotidiano 30\%, trabajo extraclase $10 \%$, una prueba $25 \%$, proyecto $25 \%$, concepto $5 \%$ y asistencia $5 \%$ (MEP, 2013b).

El cambio más significativo en la forma de evaluación fue dar un considerable valor porcentual al rubro de proyecto. De acuerdo con los nuevos programas, el aprendizaje basado en proyectos es una estrategia metodológica con técnicas, actividades y recursos organizados con un propósito pedagógico que sirve para el desarrollo de los contenidos curriculares (MEP, 2009a). El Reglamento de Evaluación de los Aprendizajes tampoco ofrece una definición clara de qué consiste un proyecto, las 
doi: http://dx.doi.org/10.15359/ree.19-2.17

URL: http://www.una.ac.cr/educare

CORREO: educare@una.cr

indicaciones son muy generales. Por otro lado, el artículo 24 del Reglamento indica que el proyecto puede ser un medio de trabajo extraclase y de investigación (MEP, 2009b).

Una segunda reforma parcial al reglamento de evaluación se dio por medio del decreto No34886-MEP (Sistema Costarricense de Información Jurídica [SCIJ], 2008). Las reformas parciales se aplicaron en el 2009 con el fin de reducir la repitencia y la exclusión:

- El estudiante reprobado de varias materias podrá matricular materias del siguiente nivel que no tengan requisito y que no presenten un choque de horario con las materias que debe repetir (Artículo 36).

- La evaluación de la conducta no afecte la evaluación académica. La nota de conducta y las notas académicas son independiente (Artículo 68).

Posteriormente, el 21 de enero del año 2013, el Consejo Superior de Educación (CSE) aprobó (Acuerdo 10-02-2013) una nueva reforma al Reglamento de Evaluación de los Aprendizajes para que los estudiantes de secundaria que hayan perdido una materia, luego de las pruebas de ampliación, tuvieran una tercera oportunidad. Para que el estudiante tenga derecho a esta tercera oportunidad debe cumplir los siguientes requisitos (MEP, 2013a):

- Haber asistido al menos al 80\% de las lecciones de esa asignatura durante el año.

- Haber cumplido con la presentación de todas las pruebas durante el año, así como con al menos un $80 \%$ de los trabajos extra clase.

- Haber cumplido con la presentación de los proyectos durante el año (en el caso de las asignaturas que utilizan los proyectos como instrumento central de la evaluación).

- Haber presentado las pruebas de ampliación en ambas convocatorias. (p. 2)

Los Comités de Evaluación de cada centro educativo son los encargados de aplicar esta nueva estrategia de promoción, además, los comités indicarán a sus estudiantes la forma en que repararán la materia: una prueba o una tarea o un trabajo.

La tercera acción estratégica impulsada en el período 2006-2014 fue llevar las reformas curriculares más allá del aula, aprovechando los espacios del centro educativo para la convivencia y la recreación. Para tal fin se creó en el año 2011 la Dirección de Vida Estudiantil (DVE), dependiente del Ministerio Académico del Ministerio de Educación Pública, como órgano responsable de planificar, coordinar, ejecutar, evaluar y supervisar programas y proyectos relacionados con el desarrollo integral del estudiantado (Poder Ejecutivo, 2014). Esta dirección se encargó de agrupar y coordinar todos los programas extracurriculares: Festival Estudiantil de las Artes, Gobiernos Estudiantiles, Juegos Deportivos Estudiantiles, Programa Bandera Azul Ecológica, Servicio Comunal y Programa Convivir (MEP, 2014). 
El Programa Convivir o Programa Nacional de Convivencia inició el 19 de septiembre del 2011 y tuvo como antecedente el proyecto "El Cole en Nuestras Manos", un plan piloto nacido en el 2007 para disminuir la deserción escolar y la búsqueda de la promoción de una cultura de paz en los centros educativos (MEP, 2014). En este proyecto participaron 64 colegios. Cada uno tuvo un comité institucional que era el encargado de elaborar una estrategia para la permanencia de sus estudiantes. Las estrategias terminaron siendo actividades extracurriculares: actividades culturales, actividades deportivas y capacitaciones (MEP, 2010).

\section{El modelo de participación estudiantil}

Las actividades extracurriculares así como las reformas curriculares buscan dar un papel más participativo al estudiantado, tanto dentro como fuera del aula. Ambas acciones tienen como supuesto un modelo que el autor Finn (1993) ha llamado modelo de participación-acción. Según Finn (1993), el estudiante que se identifica tiene desarrollado un sentido de pertenencia y el colegio constituye una parte importante de la propia experiencia. Esto conlleva, de forma secundaria, mejores logros académicos.

El segundo componente del modelo es la participación. Participar de forma activa en clase es la condición mínima para que se dé el aprendizaje formal, pues el estudiantado debe poner atención a su profesor o profesora, leer, estudiar, memorizar, responder preguntas y terminar las tareas (Finn, 1993). Considera que con estos dos componentes (identificación y participación) se crea un "círculo de desarrollo" que refuerza en el estudiantado terminar los estudios y tener éxito académico (ver figura 1).

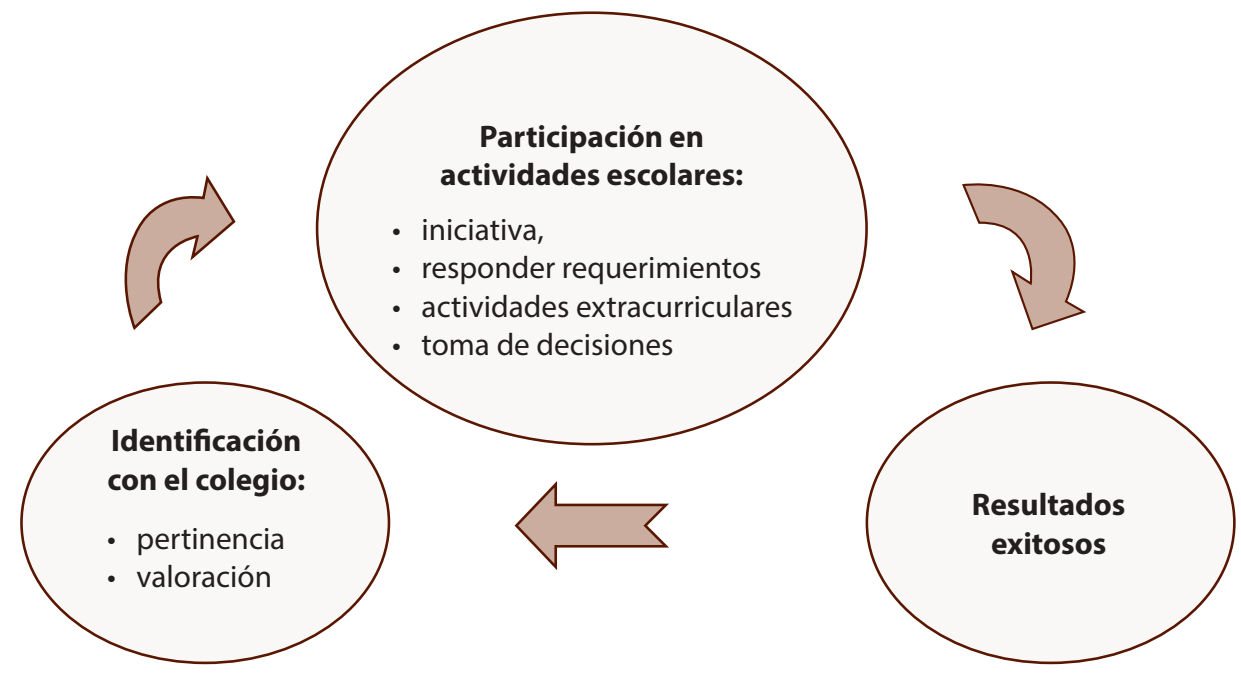

Figura 1. Elaboación propia a partir del modelo de participación-identificación de (Finn, 1993). 
doi: http://dx.doi.org/10.15359/ree.19-2.17

URL: http://www.una.ac.cr/educare

CORREO: educare@una.cr

El modelo fue validado por medio de una muestra estadística de estudiantes de octavo año de Estados Unidos. De acuerdo con los resultados se demuestra una relación fuerte entre la participación de estudiantes con los logros académicos. Estudiantes con éxitoso suelen llegar a tiempo, participan en las actividades de clase y fuera de ella. Sin embargo, los resultados fueron débiles en el vínculo entre la identificación de estudiantes y el rendimiento académico (Finn, 1993).

\section{Resultados preliminares de las acciones}

Hasta el momento, las reformas curriculares de Ética, Estética y Ciudadanía no han sido evaluadas por el Ministerio de Educación Pública. Pero un informe de la Contraloría General de la República (CGR) (2011) evaluó diez acciones implementadas por el MEP para mejorar la cobertura en educación secundaria. Entre estas diez acciones se preguntó acerca de los nuevos programas para secundaria. Del estudiantado encuestado, $52,7 \%$ consideró que lo motivaban mucho o demasiado los cambios en Educación Física, un $49 \%$ respondió lo mismo para Educación Cívica. Por otra parte, un 52,7\% y un 47,4\% de estudiantes consideró que les motivaba poco o casi nada las clases de Educación Musical y Artes Plásticas, respectivamente.

Un 60,1\% de docentes expresó poseer un conocimiento bajo o muy bajo de los nuevos programas, aunque un porcentaje casi igual 59,2\% avaló que la reforma Ética, Estética y Ciudadanía mejoró los aprendizajes del estudiantado. Al ser preguntado el personal docente si con estas reformas mejoraron la convivencia social a fin de reducir los niveles de abandono escolar en secundaria y la violencia en los centros educativos, la gran mayoría respondió que casi nada o poco (CGR, 2011).

Respecto a las reformas parciales al Reglamento de Evaluación de los Aprendizajes referente al adelantamiento de materia, la investigación hecha por la Contraloría General de la República evidenció que la capacidad instalada de los centros educativos es baja o muy baja para cumplir con lo requerido. Además, esta reforma no cuenta con la aprobación de los docentes, ya que un $74,2 \%$ manifestó que debería eliminarse por completo. El estudiantado tuvo opiniones encontradas, la mitad lo apoyaba y la otra mitad no. Sin embargo, se notó la existencia de más opinión positiva en los colegios pequeños y más negativa en los colegios grandes. Entre los principales inconvenientes señalados para implementar el adelantamiento de materias se encontró (CGR, 2011): las aulas son escasas, los horarios son cansados e incómodos, no es posible matricular todas las materias que se desean instalar, estudiantes andan por los pasillos sin hacer nada o no asisten a clases, hay desmotivación y conformismo en el estudiantado.

El Ministerio de Educación Pública, a través del Departamento de Estudios e Investigación Educativa, también realizó un estudio exploratorio sobre este tema en el año 2013. La investigación se hizo en cuatro centros educativos: dos colegios urbanos, un colegio técnico profesional y un académico rural. De acuerdo con las entrevistas y los grupos focales, el estudiantado consideró 
positivo, en general, el mecanismo de adelantamiento, pero no lo aprovecharon. Las principales razones por las que le va mal son el bajo rendimiento, los problemas de aprendizaje, problemas emocionales y de conducta y, en menor medida, razones económicas (Segura, Amador, Díaz y Gaete, 2013). Además, el adelantamiento de materias genera una carga académica adicional, pues el estudiantado lleva materias que repite y materias que adelanta, por eso, algunos terminan abandonando las materias que adelantan. Finalmente, los grupos de estudiantes expresaron que los problemas de gestión institucional generan que deambulen en el centro educativo.

Persiste la opinión negativa de docentes sobre el mecanismo de adelantamiento, por razones muy variadas: poca exigencia, deambulación de estudiantes, hay deserción de las materias, hay choque de horarios, no hay docentes tutores (Segura et al., 2013).

La tercera acción estratégica avaluada tiene que ver con las actividades extracurriculares como medios para fomentar la participación e identificación estudiantil. Para ello se revisaron dos estudios: el informe de la CGR (2011) y una investigación hecha sobre este tema por el Departamento de Estudios e Investigación Educativa del 2013.

Elinforme de laCGR(2011) esmuy breveenla evaluación delas actividades extracurriculares. Se señala que estas suelen darse más en las zonas rurales y menos en los colegios nocturnos del país. La mitad de estudiantes considera que motivan "nada o muy poco" para permanecer en el centro educativo, aunque sí considera el 75,3\% de estudiantes que beneficia su aprendizaje.

Igualmente, el Departamento de Estudios e Investigación Educativa hizo una investigación sobre la participación de estudiantes en el Festival Estudiantil de las Artes (FEA), en los Gobiernos Estudiantiles (GE), los Juegos Deportivos Estudiantiles (JDE) y el Programa Convivir (PC).

Los datos recopilados muestran que en los últimos años se ha dado un crecimiento significativo de participación estudiantil en las 27 Direcciones Regionales Educativas a la FEA. Esto se acompañó con la entrega de 1523 instrumentos musicales a 169 instituciones educativas. La mayoría de estudiantes entrevistados en la investigación opinó estar satisfecha o muy satisfecha con las actividades en general (información, organización, lugar). Igualmente la mayoría de estudiantes estaba dispuesta a seguir participando (Chavarría et al., 2013).

Respecto a los Gobierno Estudiantiles, la Dirección de Vida Estudiantil (DVE) fomentó la participación de talleres y capacitaciones para docentes y estudiantes. Entre las muchas acciones realizadas pueden mencionarse: la alianza con el Tribunal Supremo de Elecciones para fortalecer los procesos electorales estudiantiles, la publicación en el 2009 del Código Electoral Estudiantil y Reglamento para la Organización y Funcionamiento del Gobierno Estudiantil, Foros de Participación Política Estudiantil. En secundaria, la mayoría de los centros consultados para el estudio realizaron procesos electorales (96,8\%). Además la totalidad de las instituciones tienen Asambleas de Representantes oficialmente conformadas. 
doi: http://dx.doi.org/10.15359/ree.19-2.17

URL: http://www.una.ac.cr/educare

CORREO: educare@una.cr

Los Juegos Deportivos Estudiantiles (JDE), iniciados en el 1983, no ha reportado mucho cambio de acuerdo con el estudio. En el 2010 se realizaron evaluaciones a diversos aspectos de los JDE, en el 2011 se hicieron capacitaciones a promotores deportivos y representantes en las direcciones regionales de varias disciplinas deportivas. La investigación menciona como logros de JDE la alianza con del ICODER, el apoyo de algunas federaciones deportivas, entre otros.

En lo referente al Programa Convivir, se indica que la DVE en el 2012 realizó una encuesta que muestra una disparidad en la extensión del programa (ver tabla 1):

Tabla 1

Principales resultados de la encuesta del Programa Convivir 2012

\begin{tabular}{lccc}
\hline \multicolumn{1}{c}{ Aspectos evaluados } & Secundarias diurnas & Colegios técnicos profesionales & Telesecundaria \\
\hline $\begin{array}{l}\text { Cuentan con comités para la } \\
\text { promoción de la convivencia }\end{array}$ & $68,9 \%$ & $57,1 \%$ & $66,7 \%$ \\
$\begin{array}{l}\text { Cuentan con diagnósticos } \\
\text { de necesidades en materia } \\
\text { de convivencia }\end{array}$ & $46 \%$ & $71,4 \%$ & $16,7 \%$ \\
$\begin{array}{l}\text { Cuentan con planes de } \\
\text { convivencia institucional }\end{array}$ & $62,7 \%$ & $80,0 \%$ & $37,5 \%$ \\
$\begin{array}{l}\text { Han efectuado actividades } \\
\text { en esta materia }\end{array}$ & $69,4 \%$ & $80 \%$ & $25 \%$ \\
$\begin{array}{l}\text { Nivel de participación } \\
\text { estudiantil }\end{array}$ & $68,9 \%$ & $80 \%$ & $65 \%$ \\
\hline
\end{tabular}

Nota: Información tomada de la encuesta de la Dirección de Vida Estudiantil, propiedad del Departamento de Estudios e Investigación Educativa del Ministerio de Educación Pública (DVE, 2012, citado por Chavarría et al. (2013).

\section{Nuevo modelo de participación estudiantil}

En las últimas dos décadas existe un creciente interés por el concepto de engagement como un antídoto contra la alienación estudiantil en la bibliografía anglosajona. Lo estudios empíricos han dado forma a dos concepciones del tema: como una estrategia para mejorar el rendimiento académico y una forma de evaluación independiente de los resultados escolares (Dunleavy y Milton, 2011). De acuerdo con Fredricks, Blumenfeld y Paris (2004), existen tres grandes categorías de aplicación del concepto: el que se relaciona con el comportamiento, es decir, con la participación en actividades académicas y extracurriculares 
que se consideran importantes para alcanzar logros académicos positivos; el engagement emocional, que toma en cuenta las actitudes y sentimientos hacia docentes y la institución; finalmente, el cognitivo, que implica la voluntad y el esfuerzo necesario para comprender ideas complejas y dominar habilidades.

El engagement de comportamiento se llama así al relacionado con una conducta positiva que esté de acuerdo con las normas y favorezca el aprendizaje. Esto incluye tareas de aprendizaje y comportamientos como el esfuerzo, la perseverancia, la concentración, la atención, el formular preguntas y contribuir a la discusión en la clase, completar el trabajo en clase y seguir las reglas, y también, conductas negativas que indican frecuencia de tardías y ausencias (Fredricks et al., 2004). Por su parte, los autores Dotterer, McHalen y Crouter (2007) recogen los siguientes comportamientos relacionados con la participación escolar: completar la tarea, poner atención, asistir a clase y resultados académicos.

El compromiso emocional incluye las reacciones del estudiantado hacia la clase como su interés, alegría, aburrimiento, tristeza y ansiedad. Incluye su identificación con su escuela y el sentido de pertenencia. El engagement cognitivo incluye el esfuerzo directo por aprender, entender, mejorar las habilidades y el trabajo. También se incluyen habilidades para planificar estrategias de aprendizaje.

Dunleavy y Milton (2011), del Canadian Education Association identifican, por su parte, tres dimensiones para comprender el engagement: la social, la académica y la intelectual. La dimensión social tiene que ver con la participación en la vida colegial: equipos, clubes, gobierno estudiantil, campañas. La dimensión académica se refiere a la participación del estudiantado para lograr el éxito escolar y su relación con el currículo oficial, el desempeño, el apoyo de docentes y padres y madres de familia. La dimensión intelectual se ve cuando hay un compromiso psicológico y cognitivo del estudiantado, pues explora, entiende los conceptos, desarrolla ideas y resuelve problemas.

La ventaja de abordar desde estas tres dimensiones el concepto es dar una visión más dinámica y compleja del fenómeno y dejar atrás una perspectiva más individual, donde el nivel de compromiso estudiantil se determinaba por las características de este y su contexto personal (Dunleavy y Milton, 2011). En cambio, con este enfoque se toman en cuenta factores como el género, la condición étnica y económica.

Dada la riqueza académica y la visión integral sobre los factores que influyen en el desarrollo del compromiso del estudiantado hacia el aprendizaje, es recomendable adoptar como marco de referencia este concepto a fin de evaluar las estrategias de permanencia estudiantil. 
doi: http://dx.doi.org/10.15359/ree.19-2.17

URL: http://www.una.ac.cr/educare

CORREO: educare@una.cr

\section{Propuesta de tres niveles de acción preventiva}

Además de una nueva concepción sobre cómo vincular al estudiantado con su centro educativo y con sus estudios, es necesario un cambio en la forma de articular las acciones a fin de asegurar la permanencia escolar de sus estudiantes en los centros educativos. Para ello se propone trabajar un modelo preventivo basado en una pirámide invertida (ver figura 2).

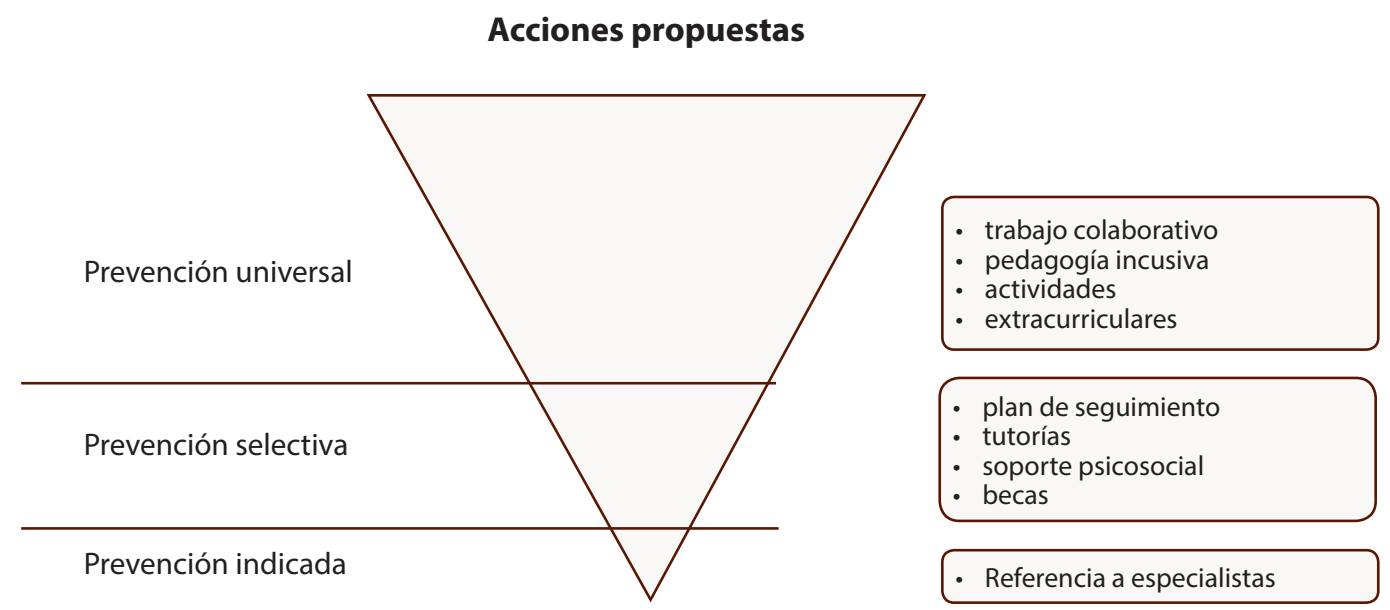

Figura 2. Modelo de pirámide invertida para las acciones de prevención del abandono escolar. Elaboración propia.

El modelo de pirámide invertida aquí propuesto se inspira en el trabajo del Response to Intervention (RTI) del American Institutes of Research introducido en los años noventa como una nueva forma de intervención para estudiantes con discapacidad (Hatch, 2014). Un modelo similar también es aplicado por la Asociación Americana de Consejeros de Colegios (ASCA, en inglés). La originalidad de lo que se propone es aplicarlo para la prevención de la deserción escolar en el contexto costarricense. La explicación que se da no busca ser exhaustiva, sino pretende dar una visión general de cómo funciona.

La pirámide invertida parte de la visión del abandono escolar como un proceso y no un evento. Es decir, se trabaja en base a indicadores que permiten monitorear y dar seguimiento a estudiantes de acuerdo con los factores de riesgo que se han definido previamente. Según estos indicadores, se establecen tres niveles de acciones preventivas. A continuación se desarrollan cuatro indicadores preliminares para elaborar la propuesta. 
De acuerdo con los sistemas preventivos desarrollados en EEUU, en cuanto a los factores de riesgo más señalados sobre abandono escolar existen tres indicadores básicos a tomar en cuenta: asistencia, bajo rendimiento, conductas de riesgo (Bruce, Bridgeland, Fox y Balfanz, 2011). También puede añadirse un cuarto indicador que es la extraedad, algo que la Unesco (2012) señala en el perfil de estudiantes que abandonan los estudios.

Las acciones preventivas universales son las más generales y están dirigidas a la totalidad de estudiantes del centro educativo. Califican en este tipo de acciones los espacios que tiene el estudiantado para desarrollarse y para participar: festivales deportivos y culturales, gobiernos estudiantiles. Tiene que ver con asegurar un ambiente seguro dentro de la institución, prácticas educativas en las aulas motivadoras y participativas.

Las acciones preventivas selectivas están dirigidas para estudiantes que presentan las primeras señas de riesgo de acuerdo con los cuatro indicadores que anteriormente fueron señalados: el ausentismo (tardías, pérdida de lecciones), bajo rendimiento (repitencia, reprobación, bajas expectativas educativas), conductas de riesgo (drogas, pandillas, embarazo adolescente, matonismo) y extraedad (tener más edad de la que corresponde a su nivel). Para esta población se diseñan acciones como el seguimiento por parte de docentes guías, tutorías académicas para estudiantes que van mal, reunión con los padres de familia, visita al hogar, apoyo para el trámite de una beca, entre otros.

Aquellos grupos de estudiantes que presentan las señales de riesgo y que no logran mejorar, a pesar de la implementación de las acciones selectivas del nivel anterior, califican para una intervención más especializada, individual y dirigida (acciones preventivas indicadas). Entre las acciones que se pueden implementar está el diagnóstico de algún tipo de problema de aprendizaje con el auxilio de especialistas, la intervención de autoridades competentes para solucionar diferentes tipos de problemas (PANI, IAFA, CCSS, CENAREC). Estas acciones responden a necesidades muy específicas de estudiantes y deben ser tratados de manera personalizada.

Este modelo requiere que haya una participación integral de todos los miembros de la comunidad educativa, que se establezcan los mecanismos institucionales para la observación y seguimiento de estudiantes de acuerdo con indicadores. Una vez definidos estos indicadores, se deben establecer los protocolos de cómo atenderles de acuerdo con los niveles de intervención aquí propuestos. La ventaja que tiene la estrategia de pirámide invertida es que crea un filtro para atenderles según sus necesidades; además, racionaliza los recursos y las acciones.

\section{Consideraciones finales}

Las tres acciones estratégicas implementadas para la reducción del abandono escolar por el Ministerio de Educación Pública (2006-2014) se ha fundamentado en propiciar la participaciónidentificación del estudiantado. Sin embargo, no hay suficiente evidencia que indique que este 
doi: http://dx.doi.org/10.15359/ree.19-2.17

URL: http://www.una.ac.cr/educare

CORREO: educare@una.cr

modelo está teniendo resultados positivos para la permanencia de estudiantes en secundaria. Las reformas curriculares están pendientes de ser evaluadas para ver si han surtido impacto en la comunidad educativa. Las reformas al Reglamento de Evaluación de los Aprendizajes, referente al adelantamiento de materias, tiene problemas de gestión y la opinión de docentes es desfavorable. Las diversas actividades extracurriculares no presentan igual desarrollo en las diversas modalidades educativas; sin embargo, el Festival Estudiantil de las Artes y los Gobiernos Estudiantiles son lo que mejor nivel de participación e identificación presentan entre los grupos de estudiantes.

Estas tres acciones están dirigidas a toda la población estudiantil, por tanto, son acciones de carácter universal. Esto impide que haya una atención más selectiva de estudiantes que presentan señales de riesgo de abandono escolar. Por esta razón, se propone el concepto de engagement como marco de referencia para una visión más integral de cómo trabajar su identificación con su centro educativo y su compromiso educativo. Igualmente, se recomienda utilizar el modelo de pirámide invertida para planificar acciones de acuerdo con las necesidades de jóvenes en riesgo. Para ello, es necesario identificar indicadores de riesgo ya mostrados en diversos estudios. La principal ventaja de este nuevo modelo es entender la deserción estudiantil o abandono escolar como un proceso y no como un evento.

\section{Referencias}

Bruce, M., Bridgeland, J. M., Fox, J. H. y Balfanz, R. (2011). On Track for Success: The Use of Early Warning Indicator and Intervention Systems to Build a Grad Nation [En camino al éxito: El uso de indicadores dealerta tempranay de sistemas de intervención para construirunanación graduada]. Recuperado de http://new.every1graduates.org/wp-content/uploads/2012/03/on track for success.pdf

Chavarría, D., Escalante, C. y Vargas, L., (2013). Principales alcances de las actividades educativas complementarias relacionadas con el Festival Estudiantil de las Artes, Gobiernos Estudiantiles, Juegos Deportivos Estudiantiles y Programa Convivencia: Un estudio de caso en centros educativos de primaria y secundaria. San José, Costa Rica: MEP, Departamento de Estudios e Investigación Educativa.

Contraloría General de la República (CGR). (2011). Informe sobre el resultado de las acciones públicas implementadas en el Ministerio de Educación Pública para mejorar la cobertura en educación secundaria (Informe No DFOE-SOC-IF-13-2011). San José, Costa Rica: División de Fiscalización Operativa y Evaluativa. Recuperado de http://www.asamblea.go.cr/Informes de la Contraloria/Informes\%202011/k-\%20informes noviembre 2011/DFOE-SOC-IF-13-2011.pdf

Dotterer. A. M., McHale, S. M. y Crouter, A. C. (2007). Implications of Out-of-School Activities and School Engagement in African American Adolescents [Consecuencias de las actividades fuera de clase y la participación escolar en adolescentes afro americanos]. Journal of Youth and Adolescence, 36, 391-401. doi: http://dx.doi.org/10.1007/s10964-006-9161-3 
Dunleavy, J. y Milton, P. (2011). Student Engagement for Effective Teaching ad Deep Learning [El compromiso del estudiante para la enseñanza efectiva y el aprendizaje profundo]. Education Canada, 48(5), 4-8. Recuperado de http://www.cea-ace.ca/sites/default/files/ EdCan-2008-v48-n5-Dunleavy.pdf

Finn, J. D. (1993). School Engagement \& Students at Risk [Compromiso escolar y estudiantes en riesgo]. Washington: National Center for Education Statistics. Recuperado de http://nces. ed.gov/pubs93/93470a.pdf

Fredricks, J. A., Blumenfeld, P. C. y Paris, A. H. (2004, primavera). School Engagement: Potential of the Concept, State of the Evidence [Compromiso escolar: Potencial del concepto, estado de la evidencia]. Review of Educational Research, 74(1), 59-109. doi: http://dx.doi. org/10.3102/00346543074001059

Hatch, T. (2014). The Use of Data in School Counseling: Hatching Results for Students, Programs and the Profession [El uso de datos en la orientación escolar: Abriendo resultados para estudiantes, programas y la profesión]. USA: Corwin.

Jiménez, W. y Gaete, M. (Enero-abril, 2013). Estudio de la exclusión educativa y abandono en la enseñanza secundaria en algunas instituciones públicas de Costa Rica. Revista Electrónica Educare, 17(1), 105-128. Recuperado de http://www.revistas.una.ac.cr/index.php/ EDUCARE/article/view/4975

Ministerio de Educación Pública (MEP). Departamento de Análisis Estadístico. (2014). Deserción intra-anual en educación regular. Recuperado de http://www.mep.go.cr/indicadores edu/ cifras desercion Intra Anual.html

Ministerio de Educación Pública (MEP). (2009a). Programas de estudio educación física: Tercer ciclo de educación general básica y educación diversificada. San José, Costa Rica: Autor. Recuperado de http://www.mep.go.cr/sites/default/files/descargas/programas-deestudio/educfisica3cicloydiversificada.pdf

Ministerio de Educación Pública (MEP). (2009b). Reglamento de evaluación de los aprendizajes. San José, Costa Rica: Autor Recuperado de http://www.mep.go.cr/educatico/reglamentode-evaluacion-de-los-aprendizajes-2009

Ministerio de Educación Pública (MEP). (2010). Proyecto el cole en nuestras manos (Informe interno). San José, Costa Rica. Manuscrito inédito.

Ministerio de Educación Pública (MEP). (2013a). Circular DM-009-02-2013. San José, Costa Rica. Recuperado de http://www.mep.go.cr/sites/default/files/pregunta frecuente/ documentos/circular dm 00902 2013.pdf 
doi: http://dx.doi.org/10.15359/ree.19-2.17

URL: http://www.una.ac.cr/educare

CORREO: educare@una.cr

Ministerio de Educación Pública (MEP). (2013b). Programa de Estudios de Artes Industriales Tercer Ciclo de Educación General Básica. San José, Costa Rica: Autor. Recuperado de http://www. mep.go.cr/sites/default/files/blog/ajduntos/programas-artes-industriales.pdf.

Ministerio de Educación Pública (MEP). (2014). Memoria institucional 2006-2014. La educación subversiva: Atreverse a construir el país que queremos. Recuperado de http://www.mep. go.cr/sites/default/files/page/adjuntos/memoriainstitucional20062014pdf.pdf

Poder Ejecutivo. (Jueves 13 de febrero, 2014). Decreto Ejecutivo No38170-MEP. La Gaceta Diario Oficial $N^{\circ} 31$. Recuperado de http://www.pgrweb.go.cr/scij/Busqueda/Normativa/ Normas $/ \mathrm{nrm}$ texto completo.aspx?param $1=$ NRTC\&nValor $1=1 \&$ nValor $2=76554 \&$ nValor 3 $=95625 \&$ strTipM $=$ TC

Programa Estado de la Nación. (2013). Cuarto informe estado de la educación. San José, Costa Rica: Autor. Recuperado de http://www.estadonacion.or.cr/estado-educacion/educacioninforme-ultimo

Sistema Costarricense de Información Jurídica (SCIJ). (Noviembre, 2008). Reforma integral de las normas reguladoras de la promoción y repitencia dentro del sistemaeducativo público costarricense (Decreto N³1635-MEP, reglamento de evaluación de los aprendizajes). N 34886-MEP. Recuperado de http://www.pgrweb.go.cr/scij/Busqueda/Normativa/Normas/nrm texto completo.aspx?p aram $1=$ NRTC\&nValor $1=1 \&$ nValor $2=64393 \&$ nValor $3=77073 \&$ strTipM $=$ TC

Segura, F., Amador. M., Díaz, Y. y Gaete, M. (2013). Estudio exploratorio: Opiniones sobre cambio en las Normas de Evaluación de los Aprendizajes, con énfasis en la opinión entorno al adelantamiento de asignaturas. San José, Costa Rica: MEP.

Unesco. (2012). Compendio mundial de educación. Oportunidades perdidas: El impacto de la repetición y de la salida prematura de la escuela. Montreal: Instituto de Estadística de la UNESCO. Recuperado de http://www.uis.unesco.org/Education/Documents/ged-2012-sp.pdf

Unicef. (2012). Completar la escuela: Un derecho para crecer un deber para compartir. Recuperado de http://www.unicef.org/lac/Datos Claves Completar la Escuela.pdf

\section{Cómo citar este artículo en APA:}

Portillo-Torres, M. C. (Mayo-agosto, 2015). Propuesta de un nuevo enfoque para reducir el abandono escolar en secundaria. Revista Electrónica Educare, 19(2), 303-316. doi: http://dx.doi.org/10.15359/ree.19-2.17

Nota: Para citar este artículo en otros sistemas puede consultar el hipervínculo "Como citar el artículo" en la barra derecha de nuestro sitio web: http://www.revistas.una.ac.cr/index.php/EDUCARE/index 